\title{
On the origin of transversal current in double-layer heterostructures
}

\author{
S.V. Iordanski \\ Landau Institute for Theoretical Physics RAS, Chernogolovka 142432, Russia \\ E-mail: iordansk@itp.ac.ru
}

Received May 11, 2016, revised June 14, 2016, published online November 25, 2016

\begin{abstract}
It is shown that usually used theoretical model for double-layer heterostructures as a pseudospin ferromagnet does not explain the observed two-dimensional spectrum. Its existence is possible when neglecting Coulomb interaction destroying two-dimensional structures and can be realized only in a strong magnetic field. That is connected also with the plain vortex lattices forming in strong magnetic fields due to thermodynamic instability. This model gives reasonable explanations of various observed effects depending on the filling of the corresponding bands. In particular in this work we show that in double-layer heterostructures a large interlayer conductance really observed can exist.
\end{abstract}

PACS: 73.21.-b Electron states and collective excitations in multilayers, quantum wells, mesoscopic, and nanoscale systems;

67.10.-j Quantum fluids: general properties.

Keywords: double-layer heterostructures, two-dimensional spectrum, magnetic fields.

The experiments with double-layer heterostructures have a long history beginning from the late eighties of the previous century. The main number of theoretical works [1,2] used the layer index as an additional quasi-spin index. However the experimental work [3] showed that the theoretical results based on this assumption contradict to the performed experiment and require some other description close to the used for one-layer systems [4].

We take the coordinate $z$ perpendicular to the plain of the layers and the coordinates $\mathbf{r}=(x, y)$ describe a twodimensional space with a constant electron potential energy $U(z)=U(-z)$ (see Fig. 1). We assume the existence of a constant magnetic field $B$ directed along $z$ large enough to make the energy of electron Coulomb interaction proportional to $\sqrt{B}$ small compared to the magnetic energy proportional to $B$. That is very important because Coulomb interaction tends to destroy two-dimensional effects. Further we shall neglect Coulomb electron interaction assuming large enough magnetic field.

It was shown in the theoretical work [5] for this case the situation is unstable and the creation of plain vortex lattices gives the thermodynamic gain. That is the case of the separate action of the variables $z$ and $\mathbf{r}$, therefore electron wave functions are the products of two functions $Z(z) \Phi(\mathbf{r})$. Various types of the vortex lattices are possible. The electron filling of the proper bands defines the physical proper- ties. The separation of the variables gives two Shrödinger equations

$$
i \hbar \frac{\partial Z}{\partial t}=-\frac{\hbar^{2}}{2 m_{e}} \frac{\partial^{2} Z}{\partial z^{2}}+U(z) Z(z)
$$

where $U(z)$ is the two-well potential (see Fig. 1).

The functions $\Phi(\mathbf{r})$ are defined by two-dimensional equation

$$
i \hbar \frac{\partial \Phi(\mathbf{r})}{\partial t}=\frac{1}{2 m_{e}}\left(-i \hbar \frac{\partial}{\partial \mathbf{r}}-\frac{e}{c} \mathbf{A}_{\mathrm{eff}}(\mathbf{r})\right)^{2} \Phi(\mathbf{r}) .
$$

Here the effective vector potential $\mathbf{A}_{\text {eff }}$ includes not only the vector potential of the external magnetic field $(1 / 2)[\mathbf{B r}]$ but also the contribution of vortices (with the

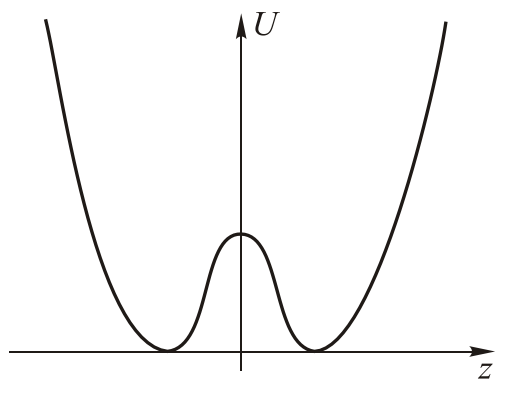

Fig. 1. Interwell potential. 
lowest energy) with the minimal circulation $2 \pi$ in each unit cell. The solutions of the Eq. (2) have simple band energy spectrum only at the rational values of the total magnetic flux through the unit cell area of the vortex lattice (see, e.g., [6]). Having in mind the experiments [7] where the electron density was close to that at the half filling of Landau level, we assume two vortices in each unit cell. Therefore the total magnetic flux through the area of the unit cell $\sigma$ will be $B \sigma-2 \Phi_{0}=(l / n) \Phi_{0}$, where $\Phi_{0}=2 \pi \hbar c /|e|$ is the flux quantum and $l, n$ are coprime integers. We see that the half filling of Landau level is achieved at $l=0$, and the steadystate solutions correspond to the representation of Abelian group of the periodic translations with the unit cell area

$$
\sigma=\frac{2 \Phi_{0}}{B}=\frac{4 \pi c \hbar}{|e| B}
$$

The situation is similar to that of graphene. It was shown in the paper [5] that the lattice have the hexagonal symmetry which is determined by the vortex lattices with the periodic vector potential in the plain $\mathbf{r}$. Having in mind the experiments [7] we assume that the vortex lattice has the hexagonal symmetry with two vortices of the minimal circulation $2 \pi$ in each elementary zone. It was shown in [5] that in this case there are two nonequivalent points $\mathbf{k}_{0}$ and $\mathbf{k}_{0}^{\prime}$ on the boundary of the two-dimensional Brillouin cell where the representation of the space group for the vortex lattice is two-fold and the full filling of the lowest band corresponds to the density of the half filled Landau level. We assume that the maximal energy in the lowest band corresponds to the energy $\varepsilon\left(\mathbf{k}_{\mathbf{0}}\right)=\varepsilon\left(\mathbf{k}_{0}^{\prime}\right)$ at the critical points. We have to add to this energy the lowest energy of the size quantization $E_{1}$, corresponding to the symmetric wave function $Z_{1}(z)=Z_{1}(-z)$ and the chemical potential $\mu$ is assumed to satisfy inequality

$$
\varepsilon(\mathbf{p})+E_{1}<\mu .
$$

We have defined the filling only for the lowest level of size quantization. For the next level $E_{2}$ of size quantization with the antisymmetric wave function $Z_{2}(z)=-Z_{2}(-z)$ we will also assume that

$$
\varepsilon\left(\mathbf{p}^{\prime}\right)+E_{2}<\mu .
$$

Therefore we have two hole Fermi circles with $\varepsilon\left(p_{F}\right)=E_{1}-E_{2}$ around the critical points $\mathbf{k}_{\mathbf{0}}$, $\mathbf{k}_{0}^{\prime}$. In what further we shall consider only the case of zero temperature.

Let us consider the stationary problem without the external electric field (details can be found in [8]). Both energies $E_{1}$ and $E_{2}$ are close to the oscillation energy $E_{0}$ in one well without tunneling. The symmetry consideration of the tunneling process gives (in semiclassical approximation) two different states with the energies $E_{1}$ and $E_{2}$, where

$$
E_{2}-E_{1}=\frac{E_{0}}{\pi} \exp \left(-\frac{1}{\hbar} \int_{-a}^{a}|p| d z\right)
$$

where $-a$ and $a$ are the positions of the turning points, the integral is in the classically forbidden region, $|p|=m_{e} v=\sqrt{2 m_{e}\left(U-E_{0}\right)}$. Therefore $E_{2}$ and $E_{1}$ are exponentially close.

In the presence of the external electric field there is an additional term in the Hamiltonian

$$
\delta \hat{H}=-|e| \int \gamma z \hat{\rho} d z d^{2} r
$$

where $\hat{\rho}$ is the operator of the electron density. We choose the electric field $\gamma>0$. Therefore we will have the current from the left well to the right one. There is no possibility to avoid the classically forbidden region. Thus we have to consider the subbarrier current. The oscillations in the left well with the energy $E_{0}$ give the ingoing wave at the beginning of the classically forbidden region

$$
\psi_{\text {in }}=\sqrt{\frac{E_{0}}{2 \pi \hbar v}} \exp \left(\frac{i}{\hbar} \int_{b}^{-a} p d z-\frac{i \pi}{4}\right) .
$$

At the point $z=a$ we get the outgoing wave in the right well $\psi_{\text {out }}=t(E) \psi_{\text {in }}$ where $t(E)$ is the transmission amplitude, $|t(E)|^{2}=D$,

$$
D=\exp \left(-\frac{2}{\hbar} \int_{-a}^{a}|p| d z\right)
$$

The electron flux to the right well (see Fig. 1) at the turning point $z=a$ is (see [8])

$$
\psi_{\text {out }}^{+} \psi_{\text {out }} v=\frac{E_{0}}{2 \pi \hbar} \exp \left(-\frac{2}{\hbar} \int_{-a}^{a}|p| d z\right) .
$$

When obtaining this formula we use only $Z(z)$ component of the electron wave function. For a more complete expression it is necessary to perform the second quantization, using the two-dimensional representation of the space group for the vortex crystal $\Phi(\mathbf{p}) \exp (\mathbf{i p r} / \hbar)$, $\Phi^{+}(\mathbf{p}) \exp (-\mathbf{p} \mathbf{p r} / \hbar)$, where $\Phi(\mathbf{p})$ is the column of two functions, $\Phi^{+}(\mathbf{p})$ is the line, mutually orthogonal and normalized (see also [5]). Therefore the full electron operators of the second quantization are

$$
\psi_{i}(t, z, \mathbf{r})=Z_{i}(z) \sum_{\mathbf{p}} \exp \left(\frac{i \mathbf{p r}}{\hbar}\right) \Phi(\mathbf{p}) a_{i}(\mathbf{p}, t)
$$

and

$$
\psi_{i}^{+}(t, z, \mathbf{r})=Z_{i}^{+}(z) \sum_{\mathbf{p}} \exp \left(\frac{-i \mathbf{p r}}{\hbar}\right) \Phi^{+}(\mathbf{p}) a_{i}^{+}(\mathbf{p}, t)
$$

here $i=1,2$ and $a_{i}(\mathbf{p}, t), a_{i}^{+}(\mathbf{p}, t)$ are the usual Fermi operators in Heisenberg representation with the energy $\varepsilon(p)<E_{1}-E_{2}+\mu$, here $\mu$ is the chemical potential equal to the total energy in Dirac points.

The quantities $Z_{i}(z), Z_{i}^{+}(z)$ do not depend on the functions $\Phi(\mathbf{p}), \Phi^{+}(\mathbf{p})$, but their number is essential for the 
counting of the different filled states. The number of the various states in the crystal band coincides with the number of the elementary cells. For the two-dimensional case it gives $N=S / \sigma$, where $S$ is the sample area, and $\sigma$ is the area of the elementary cell. Generally speaking, we should count only the filled states and exclude the states in the hole Fermi circles near Dirac points. Their quantity is exponentially small according to Eq. (6) and we neglect them.

Using Eq. (9) we get for the electron transition rates from the left well to the right well

$$
\frac{d N_{l}}{d t}=\frac{S}{\sigma} \frac{E_{0}}{2 \pi \hbar} \exp \left(-\frac{2}{\hbar} \int_{-a}^{a}|p| d z\right) .
$$

However besides this channel of the electron transitions there is also a channel of the electron transitions from the right well to the left well with the opposite direction of the current

$$
\frac{d N_{r}}{d t}=-\frac{S}{\sigma} \frac{E_{0}}{2 \pi \hbar} \exp \left(\frac{2}{\hbar} \int_{a}^{-a}|p| d z\right) .
$$

The full change of the electron number in the right well is given by a sum $d N_{t} / d t=d N_{l} / d t+d N_{r} / d t$. In the absence of the external electric field and $U(z)=U(-z)$ there is no current between the wells $d N_{t} / d t=0$. In the presence of the electric field the direction of the current coincides with the direction of the electric field and therefore

$$
\frac{d N_{t}}{d t}=\frac{d N_{l}}{d t}
$$

and we have to calculate the subbarrier current for a small value of the electric field $\gamma$. The effective potential energy is $U_{\text {eff }}(z)=U(z)-\gamma|e| z$ (see Fig. 2). The subbarrier imagine momentum at $z>0$ is given by the expression $|p|=\sqrt{2 m_{e}\left[U(z)-\gamma|e| z-E_{0}\right]}$ and we see that the electric field results in the decrease of the barrier height. Supposing the symmetry at $z \rightarrow(-z)$ one can replace $z \rightarrow|z|$ resulting in the decrease of the barrier height in the whole domain of integration. At small electric field $\gamma$ we obtain in the linear approximation

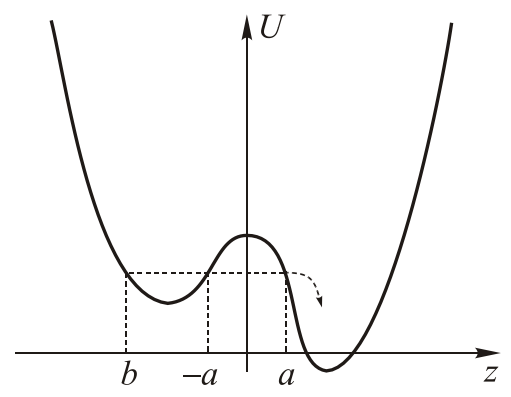

Fig. 2. The interwell potential modified by the electric.

$$
|p| \simeq \sqrt{2 m_{e}\left[U(z)-E_{0}\right]}-\frac{\gamma|e \| z| \sqrt{2 m_{e}}}{2 \sqrt{U(z)-E_{0}}}
$$

The appearance of $|z|$ instead of $z$ indicates only one direction of the subbarrier current.

Substituting this expression in the Eq. (12) in linear on $\gamma$ approximation one gets

$$
\begin{gathered}
\exp \left(-\frac{2}{\hbar} \int_{-a}^{a}|p| d z\right) \simeq \exp \left(\frac{-2}{\hbar} \int_{-a}^{a} d z \sqrt{2 m_{e}\left[U(z)-E_{0}\right]}\right) \times \\
\times\left[1+\frac{1}{\hbar} \int_{-a}^{a} d z_{1} \frac{\gamma\left|e \| z_{1}\right| \sqrt{2 m_{e}}}{\sqrt{U\left(z_{1}\right)-E_{0}}}\right] .
\end{gathered}
$$

The term without $\gamma$ does not contribute to the current and can be omitted. The turning points in the linear approximation coincide with $a$ and $-a$ in the absence of electric field. Thus we get for the net current to the right well

$$
\begin{gathered}
\frac{d N_{t}}{d t}=\frac{S}{\sigma} \frac{E_{0}}{2 \pi \hbar} \exp \left(-\frac{2}{\hbar} \int_{-a}^{a} d z \sqrt{2 m_{e}\left[U(z)-E_{0}\right]}\right) \times \\
\times \frac{1}{\hbar} \int_{-a}^{a} d z_{1} \frac{|e| \sqrt{2 m_{e} \gamma}\left|z_{1}\right|}{\sqrt{U\left(z_{1}\right)-E_{0}}} .
\end{gathered}
$$

According to definition the coefficient in front of a small electric field $\gamma$ gives the conductance of the twolayer heterostructure. As it is usual in linear response, we do not calculate the nonlinear entropy production in the right well. Let us analyze our final expression (14). Proportionality to the sample area is confirmed by the experiment [9]. The important factor $S / \sigma=S|e| B /(4 \pi c \hbar)$ which is large in a strong magnetic field and may result in a large value of the product

$$
\frac{S}{\sigma} \exp \left(-\frac{2}{\hbar} \int_{-a}^{a} d z \sqrt{2 m_{e}\left[U(z)-E_{0}\right]}\right),
$$

thus explaining the high currents observed in the experiment [7]. The other factors are strongly dependent on the semiclassical approximation used in our calculations. The considered physical problem gives an example of the conductance due to subbarrier currents.

The vortex model is the inevitable consequence of the thermodynamic instability in our system and it is connected with the magnetization in a strong magnetic field. The electric field arises due to the change of the magnetic field and it induces electric current because the static magnetic field itself can not produce the work on the electrons. Using of the vortex lattice model makes unnecessary the additional constructions like Chern-Simons field or the composite fermions. In the recent works $[10,11]$ authors use more complicate models assuming exact electron-hole symmetry. Another difficulty is connected with the observed 
electron spectrum which is far from the standard Landau levels. In the vortex model the spectrum is closely connected with the separation of the variables like in the case of one-layer structures.

Author express his gratitude to E. Kats, I. Kolokolov, and D. Khmelnitskii for the discussions. The work was supported by grant RSc\#14-12-00898.

1. New Perspectives in QHE, S. Das Sarma and A. Pinczuk (eds.), Willey, NY (1997).

2. S.M. Girvin and A.H. MacDonald, in: New Perspectives in QHE, S. Das Sarma and A. Pinczuk (eds.), Willey, NY (1997), ch. 5.

3. Stefano Luin, Vittorio Pellegrini, Aron Pinczuk, Brian S. Dennis, Loren N. Pfeiffer, and Ken W. West, Phys. Rev. Lett. 94, 146804 (2005).
4. S.V. Iordanski, Pisma v ZhETF 99, 606 (2014).

5. S.V. Iordanski and D.S. Lyubshin, J. Phys. Condens. Matter 21, 45601 (2009).

6. E.M. Lifshitz and L.P. Pitaevski, Statistical Physics, Fizmatlit, Moscow (2002), ch. 2; Pergamon, NY (1981).

7. I.B. Spielman, J.P. Eisenstein, L.N. Pfeiffer, and K.W. West, Phys. Rev. Lett. 84, 5808 (2000).

8. L.D. Landau and E.M. Lifshitz, Quantum Mechanics, Fizmatlit, Moscow (2002), Vol. 3, ch. 7; Pergamon, NY (1991).

9. K. Finck, A.R. Champagne, J.P. Eisenstein, L.N. Pfeiffer, and K.W. West, Phys. Rev. B 78, 075302 (2008).

10. C. Wang and T. Senthil, arXiv: $1507.08290 v 2$ cond.mat.str-el.

11. G. Murthy and R. Shankar, arXiv: 1508.06974v2 cond.mat.str-el. 